\title{
Super-Mini Percutaneous Nephrolithotomy in the Treatment of Pediatric Nephrolithiasis: Evaluation of the Initial Results
}

\author{
Yongda Liu, MD, ${ }^{1, *}$ Wenqi Wu, MD, PhD, ${ }^{1, *}$ Aierken Tuerxun, MD, ${ }^{2, *}$ Yang Liu, MD, ${ }^{1, *}$ Abulizi Simayi, MD, \\ Jinxing Huang, MD, Abudukahaer Batuer, MD, Yizhou Zhou, MD, Jiawei Luo, MD,' Wen Zhong, MD, \\ Zhijian Zhao, MD, Wei Zhu, MD, and Guohua Zeng, MD, PhD ${ }^{1}$
}

\begin{abstract}
Objective: To evaluate the efficacy and safety of super-mini percutaneous nephrolithotomy (SMP) in the treatment of pediatric kidney stones.

Patients and Methods: We reviewed the records of 111 children with renal stones treated with SMP technique in four different centers between September 2014 and September 2015. The indications for SMP treatment in all these kids were either previously failed shock wave lithotripsy or retrograde intrarenal surgery approaches, according to their parents' preferences. Nephrostomy tracts used in the SMP system ranged from 10F to 14F in size. Lithotripsy was performed using either a Holmium laser or pneumatic lithotripter. Perioperative and postoperative parameters along with operative data were recorded in detail and stone components were analyzed by infrared spectroscopy.

Results: This study included 71 boys and 40 girls with a mean age of $3.90 \pm 3.53$ years (range $0.5-15$ ). The mean stone burden was $1.4 \pm 0.6 \mathrm{~cm}$ (range 0.8-4.8). Mean operative time was 39.4 26.2 minutes (range 7105). The mean hemoglobin drop was $10.2 \pm 7.1 \mathrm{~g} / \mathrm{L}$ (range 0-25) and no transfusion was needed. Significant complications were observed in $17(15.3 \%)$ children with 10 and 7 cases in Clavien grade I and grade II, respectively. Complete stone clearance on postoperative day 1 and on 3-month follow-up was 84.7\% (94/111) and $90.1 \%$ (100/111), respectively. Ninety-five (85.6\%) children did not require any type of catheters (total tubeless). The mean hospital stay was $2.7 \pm 1.5$ days (range $1-7$ ).

Conclusions: Our preliminary data demonstrated that SMP was safe and effective. SMP could be a feasible treatment option for pediatric stone disease. Further randomized controlled trials are still needed to prove the efficacy of using the SMP system in children, particularly in those with larger stones.
\end{abstract}

Keywords: kidney stones, pediatric, super-mini percutaneous nephrolithotomy

\section{Introduction}

$\mathbf{P}$ EDIATRIC STONE DISEASE poses a challenge to urologists. Due to the common underlying metabolic and anatomic abnormalities, children with urolithiasis are part of the highrisk group for stone recurrence. ${ }^{1,2}$ Thus, the management of calculi in children requires a complete stone clearance to minimize the number of procedures and the detrimental effects on the growing kidneys.
Although shock wave lithotripsy (SWL) remains the firstline treatment option for most pediatric upper tract urinary calculi, ${ }^{3}$ the unpredictable results and higher retreatment rates are the main drawbacks of this approach. ${ }^{4}$ As was the concern with SWL, retrograde intrarenal surgery (RIRS) might cause vesicoureteral reflux and strictures in children, as a result of dilatation of small-caliber ureteral orifices by ureteral access sheath. ${ }^{5}$ Moreover, additional general anesthesia is required for subsequent stent removal.

\footnotetext{
${ }^{1}$ Department of Urology, Minimally Invasive Surgery Center, The First Affiliated Hospital of Guangzhou Medical University, Guangdong Key Laboratory of Urology, Guangzhou, China.

${ }^{2}$ Department of Urology, The First People's Hospital of Kashi, Xinjiang, China.

${ }^{3}$ Department of Urology, The Xinjiang Uyghur Autonomous Region People's Hospital, Xinjiang, China.

${ }^{4}$ Department of Urology, Shache County People's Hospital, Xinjiang, China.

*These authors contributed equally to this work
} 
Impacted collecting system and delicate parenchyma of the children's kidney necessitate the use of the least invasive treatment approaches to limit the extent of damaging function. ${ }^{6,7}$ Percutaneous nephrolithotomy (PCNL) is advocated as a safe and effective treatment for children with larger and more complex calculi. However, there is continued concern that it will cause long-term renal damage and scarring. ${ }^{8} \mathrm{Re}-$ cently, the miniaturization of PCNL equipment has revolutionized stone management. ${ }^{9-11}$ Accumulated evidences so far have proven that when performed in children, miniaturized PCNL techniques, including miniperc, microperc, and ultra-mini perc (UMP), were all associated with decreased morbidity and acceptable stone-free rates. ${ }^{12-14}$

The super-mini PCNL (SMP) system was first defined and introduced as an alternative treatment of urinary stones less than $2.5 \mathrm{~cm}$ by Zeng et al. ${ }^{15} \mathrm{In}$ this present study, by reporting our initial data, we evaluated the efficiency and safety of SMP technique in children with moderate-sized renal calculi.

\section{Patients and Methods}

\section{Patients}

A total of 111 children treated in four different centers between September 2014 and September 2015 were included in this study program. Institutional review board and ethics committee approval documents were obtained from each of the participating centers. Parents and children (if possible) were well informed about the technique, and informed consent was obtained from all patients (or their parents) before the procedure. The inclusion criteria for our study were (1) children with stones resistant to and/or requiring multiple sessions for SWL and RIRS treatment, (2) stone diameter larger than $2.0 \mathrm{~cm}$, or (3) those parents who preferred SMP regardless of stone size.

\section{Methods}

The detailed information regarding the patients' demographics and stone-related factors (size, location, and composition) has been evaluated and recorded. The preoperative tests included complete blood count, routine urine test, urine culture, and serum biochemistry. In patients older than 3 years, 24-hour urine sample was collected for stone risk analysis. Radiologic evaluation of the stone included kidneys, ureters, and bladder (KUB), low-dose CT, and abdominal ultrasonography (US). In patients with a positive culture, antibiotics were selected according to the bacterial sensitivity results. Patients who had negative urine cultures received a single dose of broad-spectrum antibiotic prophylaxis just before the procedure.

\section{SMP technique}

The patient was placed in the lithotomy position under general anesthesia. A $4 \mathrm{~F}$ or $5 \mathrm{~F}$ open-end ureteral catheter (Boston Scientific Corporation, Miami, FL) was retrogradely inserted into the collecting system. The patient was then turned prone with appropriate padding placed under the chest and the ipsilateral flank. The selected calix was punctured under ultrasound guidance and a 0.032 -inch guidewire was inserted into the collecting system. Nephrostomy tract was established using metal dilators (12-14F depending on patient's size and the stone burden) in one step. The corre- sponding size of suction-evacuation sheath was then placed. The sheath was connected to the specimen collection bottle via the oblique branch of a metal connector. A rubber cap, with a center aperture, was placed at the end of the straight branch of the connector. A negative pressure aspirator was attached to the collection bottle. The negative pressure was adjusted to a setting of $150-200 \mathrm{mmHg}$. The miniature endoscope was inserted into the sheath through the metal connector. The main irrigation was delivered into the working channel of the endoscope sheath using a pump. When the flow of the main irrigation system was insufficient, the auxiliary irrigation system could be used. Holmium:YAG laser with a laser fiber up to $365 \mu \mathrm{m}$ and/or pneumatic lithotripter with a $0.8 \mathrm{~mm}$ probe were used for stone fragmentation. Under continuous suction, tiny stone fragments passed through the space between the scope and the sheath and then exited through the oblique sluice. For larger fragments, the scope was slowly withdrawn to the end of the connector to form an unimpeded channel, and then, the large fragments could pass through the oblique side-port. The negative pressure could be adjusted by either partially or completely occluding the pressure vent. A basket was used for difficult aspirated fragments. The procedure for the SMP technique has been described in detail previously. ${ }^{15}$

A visual and fluoroscopic examination was performed to estimate stone-free status at the end of the procedure. "Totally tubeless PCNL" (no ureteral stent and nephrostomy tube) was performed in cases without residual stone fragment and significant bleeding. A Double-J or nephrostomy tube was only used in the presence of residual stone fragments, significant bleeding, extravasation, or upper urinary tract obstruction.

The stone-free status was evaluated by KUB and/or US or low-dose CT, if necessary, on postoperative day 1 and at 3 months. Any residual fragments less than $2 \mathrm{~mm}$ were considered to be clinically insignificant residual fragments (CIRF). A patient was considered to be stone free if there were no residual fragments or with clinically "insignificant" residual stones on radiologic evaluation after the procedure.

The chemical compositions of the stones were analyzed using infrared spectroscopy. Intraoperative and postoperative procedure-related parameters were recorded, including operational duration; number and position of accesses; need for nephrostomy tube and/or Double-J stent; complications and hemoglobin decrease. In addition, stone clearance rates and hospital stay were also analyzed in each patient.

\section{Results}

A total of 111 procedures were performed in 71 boys and 40 girls $($ male/female $=1.81)$. The mean age of the children was $3.9 \pm 3.5$ years (range $0.5-15)$ and $65(58.6 \%)$ of them were younger than 3 years. The mean stone size was $1.4 \pm$ $0.6 \mathrm{~cm}$ (range $0.8-4.8$ ) with a mean stone density value of $735.2 \pm 329.1 \mathrm{HU}$ (range 288-1378). Mean body mass index value was $17.0 \pm 3.4 \mathrm{~kg} / \mathrm{m}^{2}$ (range $11.2-31.9$ ). The characteristics of the patients are summarized in Table 1.

Of the 111 patients, 25 patients (22.5\%) had bilateral stones. All patients with bilateral urinary stones only needed unilateral SMP. The contralateral stones were small and treated by other means. A single nephrostomy tract was used in all patients. Of all the cases, $2(1.8 \%)$ tracts were $10 \mathrm{~F}$ in size, $94(84.7 \%)$ tracts were $12 \mathrm{~F}$, and $15(13.5 \%)$ tracts were $14 \mathrm{~F}$. 
Table 1. Demographics and Stone Characteristics of the Patients Who Underwent Super-Mini Percutaneous NePhrolithotomy

\begin{tabular}{lc}
\hline Characteristics & Number \\
\hline Patients, $n$ & 111 \\
Male:Female (ratio) & $71: 40(1.8)$ \\
Mean age \pm SD, years & $3.9 \pm 3.5$ \\
Range & $0.5-15$ \\
Younger than 3 years, $n(\%)$ & $65(58.6 \%)$ \\
Mean (SD, range) BMI, kg/m ${ }^{2}$ & $17.0 \pm 3.4(11.2-31.9)$ \\
Mean (SD, range) stone & $1.4 \pm 0.6(0.8-4.8)$ \\
size, cm & \\
Positive preoperative & $16(14.4 \%)$ \\
urine culture, $n(\%)$ & \\
Patient stone in kidney position, $n(\%)$ & \\
Left & $43(38.7 \%)$ \\
Right & $43(38.7 \%)$ \\
Bilateral & $25(22.5 \%)$ \\
Mean (SD, range) & \\
Hounsfield units & \\
Stone location, $n(\%)$ & 111 \\
Upper ureter & $33(29.7 \%)$ \\
Pelvis & $26(23.4 \%)$ \\
Upper calix & $14(12.6 \%)$ \\
Middle calix & $9(8.1 \%)$ \\
Lower calix & $21(18.9 \%)$ \\
Multiple & $8(7.2 \%)$ \\
Access sheath size, $n(\%)$ & 111 \\
F10 & $2(1.8 \%)$ \\
F12 & $94(84.7 \%)$ \\
F14 & $15(13.5 \%)$ \\
\hline
\end{tabular}

Intraoperative parameters are summarized in Table 2. The renal punctures were done via supracostal and infracostal approaches in $76(68.5 \%)$ and $35(31.5 \%)$ patients, respectively. Mean operative time, which was defined as the interval, from puncturing the needle to the removal of drapes was 39.4 \pm 26.2 minutes (range 7-105). The mean hemoglobin loss was 10.2 \pm $7.1 \mathrm{~g} / \mathrm{L}$ (range $0-25$ ). None of the children required blood transfusion. The complete stone clearance on postoperative day 1 was $84.7 \%$ (94/111), with $6.3 \%$ (7/111) considered as CIRF. Another $10(9.0 \%)$ cases had residual calculi of $>2 \mathrm{~mm}$. Of those children, four required an RIRS and the other six required an SWL to disintegrate the residual fragments. During a 3-month follow-up, the SFR increased to $90.1 \%$ (100/111) with $6(5.4 \%)$ children having CIRF. Five (4.5\%) had asymptomatic residual fragments of 3-6 mm. For these cases, a close follow-up program was applied.

Total tubeless SMP was applied in 95 (85.6\%) cases. Thirteen $(11.7 \%)$ children had a Double-J stent placed for $2-$ 6 weeks due to the following reasons: endopyelotomy for PUJ obstruction in 2 cases, ipsilateral distal ureteral stone with semirigid ureteroscope in 3 , significant pyelocaliceal blood clots in 1, significant residual stones in 4, and 3 cases for minor pelvis perforation. Nephrostomy tube was placed in $3(2.7 \%)$ children due to urinary extravasation.

A total of $17(15.3 \%)$ children had significant complications and were classified using the Clavien grading system. ${ }^{16}$ Postoperative fever was the most common complication observed in $7(6.3 \%)$ children. No significant difference of complications was found between children with supracostal and infracostal puncture (Supplementary Table S1; Supple-
Table 2. Intraoperative And Postoperative VARIABLES

\begin{tabular}{|c|c|}
\hline Variable & Value \\
\hline Mean operative time $( \pm S D)$, minute & $39.4 \pm 26.2(7-105)$ \\
\hline Mean hemoglobin drop $( \pm S D), g / L$ & $10.2 \pm 7.1(0-25)$ \\
\hline \multicolumn{2}{|l|}{ No. of puncture locations $(n=111)$} \\
\hline Upper pole/Interpolar/ & $65(58.6 \%) /$ \\
\hline Lower pole calix, $n(\%)$ & $\begin{array}{l}33(29.7 \%) / \\
13(11.7 \%)\end{array}$ \\
\hline Supracostal/Infracostal, $n(\%)$ & $\begin{array}{l}76(68.5 \%) / \\
35(31.5 \%)\end{array}$ \\
\hline \multicolumn{2}{|l|}{ Initial SFR, $n(\%)$} \\
\hline Completely stone free & $94(84.7 \%)$ \\
\hline Residual fragments & $10(9.0 \%)$ \\
\hline $\begin{array}{l}\text { Insignificant residual stone } \\
\text { fragments }(<2 \mathrm{~mm})\end{array}$ & $7(6.3 \%)$ \\
\hline \multicolumn{2}{|l|}{ Final SFR at 3 months, $n(\%)$} \\
\hline Completely stone free & $100(90.1 \%)$ \\
\hline Residual fragments & $5(4.5 \%)$ \\
\hline $\begin{array}{l}\text { Insignificant residual stone } \\
\text { fragments }(<2 \mathrm{~mm})\end{array}$ & $6(5.4 \%)$ \\
\hline Significant complication, $n(\%)$ & $17(15.3 \%)$ \\
\hline Fever $\left(>38.5^{\circ} \mathrm{C}\right)$ & $7(6.3 \%)$ \\
\hline Grade I & 6 \\
\hline Grade II & 1 \\
\hline Hematuria (Grade I) & $4(8.1 \%)$ \\
\hline Extravasation (Grade II) & $3(2.7 \%)$ \\
\hline Perforation (Grade II) & $3(2.7 \%)$ \\
\hline Blood transfusion rate & 0 \\
\hline Total tubeless, $n(\%)$ & $95(85.6 \%)$ \\
\hline Double-J stent, $n(\%)$ & $13(11.7 \%)$ \\
\hline Nephrostomy tube, $n(\%)$ & $3(2.7 \%)$ \\
\hline $\begin{array}{l}\text { Mean postoperative } \\
\text { hospital stay, days }\end{array}$ & $2.7 \pm 1.5(1-7)$ \\
\hline
\end{tabular}

mentary Data are available online at www.liebertpub.com/ end). Of the seven cases, two were cured by keeping the urethral catheter for 24-48 hours, one case required additional oral antibiotics, the remaining four had a spontaneous recovery without special management. Four $(3.6 \%)$ children had hematuria (Grade I), which resolved spontaneously without further intervention. Three $(2.7 \%)$ children had minor pelvis perforation (Grade II), which was managed by leaving a Double-J stent for 2 weeks. Another $3(2.7 \%)$ children with urinary extravasation (Grade II) were treated with diuretic and keeping a nephrostomy tube for 2448 hours. No other complications beyond grade III were noted. The mean length of stay (LOS) was $2.7 \pm 1.5$ days (range 1-7).

\section{Table 3. The Major Stone Compositions of Children Treated With Super-Mini Percutaneous Nephrolithotomy}

Stone composition Number, $\mathrm{n}$

Calcium phosphate

Magnesium ammonium phosphate

Carbonate apatite

Uric acid

Calcium oxalate

Ammonium urate

Cystine

Total

4

6

1

10

25

37 84 
Stone composition analyses were available in 84 patients. Ammonium urate was the most common stone composition (Table 3).

\section{Discussion}

Improvements in technology and growing experience have dramatically changed the concepts of pediatric urolithiasis management in the last two decades. ${ }^{17,18}$ Historically, large renal stones in children were treated by open surgical removal with prolonged hospital admission. This approach has largely been replaced by minimally invasive techniques, including SWL, PCNL, and RIRS methods. ${ }^{17}$ Although SWL is associated with minimal morbidity, its clearance in certain stone components such as cysteine and lower pole stones is marginal. ${ }^{19}$ Additional general anesthesia sessions and further radiation exposure may be required for multiple SWL sessions in children. In recent years, with significant improvements in the miniaturization and durability of endoscopic equipment, RIRS has become a common alternative treatment modality in young children. Nevertheless, the stone clearance of RIRS can be significantly affected by the stone burden and renal anatomy. ${ }^{5}$ Also, long-term complications of pediatric RIRS such as vesicoureteral reflux and UVJ stricture remain undefined.

The ultimate objective of stone therapy is to render the patient stone free. This is particularly crucial in the pediatric population as urolithiasis in children is often associated with anatomical and metabolic abnormalities, and the risk of complications and recurrences is high. ${ }^{1}$ In this study, the initial SFR of SMP on postoperative day 1 was $84.7 \%$, and this rate was increased to $90.1 \%$ at postoperative 3 months. Our findings are in line with other published series of miniaturized PCNL in children. ${ }^{12-14}$

The adoption of PCNL for the treatment of stone disease in children has lagged behind in adults due to concerns regarding the use of large size nephrostomy tract in a small collecting system. Accumulating evidences have suggested that the size of the tract is significantly correlated with the morbidity of PCNL in children. ${ }^{20,21}$ Bilen et $\mathrm{l}^{21}$ compared the safety of three different sizes of tract (26F, 20F, and 14F) in children and found an obvious lower rate of complications and transfusions in the 14F tract group. In our trial, most of the cases $(84.7 \%)$ were achieved with a $12 \mathrm{~F}$ sheath. The mean hemoglobin deficit was $10.2 \mathrm{~g} / \mathrm{L}$, and none of the patients required a blood transfusion.

Recent series encourage the adoption of UMP and microperc for the treatment of small renal stones in pediatric patients. $^{22}$ Silay et al ${ }^{12}$ performed the microperc in 19 children with a mean stone size of $14.8 \mathrm{~mm}$, the overall SFR at 1 month was $89.5 \%$, and the mean hemoglobin decrease was $0.1 \pm 0.3 \mathrm{mg} / \mathrm{dL}$. Dede et $\mathrm{al}^{13}$ reported their experience of UMP treatment in 39 children with a mean stone burden of $20.4 \mathrm{~mm}$. A similar SFR $(87.1 \%)$ to that of microperc was achieved with UMP. Those miniaturized PCNL techniques have been shown with minimal injury to children's kidney without reducing the effectiveness. However, due to the usage of fine tracts, drawbacks of these techniques included raised intrapelvic pressures, insufficient irrigation, and inability to retrieve larger fragments. ${ }^{9-11}$

New technique may face the question whether operative complexity, fragmentation requiring time, and the difficulty of calculi removal are increased. In the SMP procedure, however, stone fragments were actively removed by a $12 \mathrm{~F}$ or $14 \mathrm{~F}$ modified suction sheath with continuous negative pressure aspiration. Another advantage of SMP is it does not require forceps to extract fragments. Therefore, SMP is not only able to shorten the operating time but most importantly it also decreases the renal pelvic pressure. In our study, the mean operative time was $39.4 \pm 26.2$ minutes, and only seven $(6.3 \%)$ children developed a minor postoperative fever.

An optimal treatment for renal stone should ensure high stone clearance with low complications rate. SMP has been shown to be safe with a total of $15.3 \%$ complication rate in this trial, which was similar to those of UMP $(15.3 \%)^{13}$ and $16 \mathrm{~F}$ miniperc $(15.8 \%){ }^{14}$ All the complications were in Clavien grade I to II, and postoperative fever was the most common complication. No other serious complications (grade III to IV) were observed.

One of the important drawbacks of the URS is the necessity of placing a post-URS stent in all children. Stents result in symptoms, healthcare cost, decreased quality of life, and repeated anesthesia to facilitate removal. In our study, only $13(11.7 \%)$ children had a Double-J stent placement and 95 children $(85.6 \%)$ were totally tubeless. Three children had a nephrostomy tube $(2.7 \%)$. Tubeless PCNL has been shown to decrease hospital stay and postoperative pain. ${ }^{23}$ The mean LOS of our patients was $2.7 \pm 1.5$ days, this was comparable with those recent UMP procedures performed in children $(2.8 \pm 0.42$ days $) .^{13}$

All fragments remaining in the urinary tract 3 months after the last intervention were considered as residual fragments. At present, the term "clinically insignificant residual fragments" or "CIRF"' was referring to residual fragments after SWL, RIRS, or PCNL, which are smaller than $4 \mathrm{~mm}$ (or sometime $5 \mathrm{~mm}$ ), asymptomatic, nonobstructive, noninfectious, and associated with sterile urine. ${ }^{24-26}$ Evidence showed that residual stones $>6 \mathrm{~mm}$ and symptomatic stones $<4-5 \mathrm{~mm}$ are indications for active stone removal. The indication for follow-up is asymptomatic stones $<4-5 \mathrm{~mm} .^{27}$ In our study, a close follow-up program was applied for children with asymptomatic residual fragments of 3-6 mm.

We believe that each technique has its indications. SMP will not replace the URS or SWL but simply complement them for children with moderate stone size. Based on our experience, SMP could minimize the invasiveness with high efficiency compared with standard PCNL. The careful selection of patients is one of the key factors for avoiding complications and increasing the success rates.

\section{Conclusions}

Our current findings demonstrated that the SMP technique could be a safe and effective alternative for the management of pediatric kidney stones. With its tubeless feature, SMP is suitable for renal calculi that are not amenable to RIRS or SWL in pediatric cases. We imply that for pediatric stones of $10-20 \mathrm{~mm}$, SMP could be an ideal choice with a high stonefree rate and minimal invasiveness.

\section{Author Disclosure Statement}

No competing financial interests exist. 


\section{References}

1. Sarica K. Pediatric urolithiasis: Etiology, specific pathogenesis and medical treatment. Urol Res 2006;34:96-101.

2. DeFoor W, Asplin J, Jackson E, et al. Urinary metabolic evaluations in normal and stone forming children. J Urol 2006;176:1793-1796.

3. Preminger GM, Tiselius H-G, Assimos DG, et al. 2007 Guideline for the Management of Ureteral Calculi. Eur Urol 2007;52:1610-1631.

4. Srisubat A, Potisat S, Lojanapiwat B, Setthawong V, Laopaiboon M. Extracorporeal shock wave lithotripsy (ESWL) versus percutaneous nephrolithotomy (PCNL) or retrograde intrarenal surgery (RIRS) for kidney stones. Cochrane Database Syst Rev 2014;11:CD007044.

5. Resorlu B, Oguz U, Resorlu EB, Oztuna D, Unsal A. The impact of pelvicaliceal anatomy on the success of retrograde intrarenal surgery in patients with lower pole renal stones. Urology 2012;79:61-66.

6. Mor Y, Elmasry YE, Kellett MJ, Duffy PG. The role of percutaneous nephrolithotomy in the management of pediatric renal calculi. J Urol 1997;158(3 Pt 2):1319-1321.

7. Dawaba MS, Shokeir AA, Hafez A, et al. Percutaneous nephrolithotomy in children: Early and late anatomical and functional results. J Urol 2004;172:1078-1081.

8. Michel MS, Trojan L, Rassweiler JJ. Complications in percutaneous nephrolithotomy. Eur Urol 2007;51:899-906.

9. Datta SN, Solanki R, Desai J. Prospective outcomes of ultra mini percutaneous nephrolithotomy: A Consecutive Cohort Study. J Urol 2016;195:741-746.

10. Mishra S, Sharma R, Garg C, Kurien A, Sabnis R, Desai M. Prospective comparative study of miniperc and standard PNL for treatment of 1 to $2 \mathrm{~cm}$ size renal stone. BJU Int 2011;108:896-899.

11. Desai MR, Sharma R, Mishra S, Sabnis RB, Stief C, Bader M. Single-step percutaneous nephrolithotomy (microperc): The initial clinical report. J Urol 2011;186:140-145.

12. Silay MS, Tepeler A, Atis G, et al. Initial report of microperc in the treatment of pediatric nephrolithiasis. J Pediatr Surg 2013;48:1578-1583.

13. Dede O, Sancaktutar AA, Dagguli M, Utangac M, Bas O, Penbegul N. Ultra-mini-percutaneous nephrolithotomy in pediatric nephrolithiasis: Both low pressure and high efficiency. J Pediatr Urol 2015;11:253 e1-e6.

14. Zeng G, Zhao Z, Zhao Z, Yuan J, Wu W, Zhong W. Percutaneous nephrolithotomy in infants: Evaluation of a single-center experience. Urology 2012;80:408-411.

15. Zeng G, Wan S, Zhao Z, et al. Super-mini percutaneous nephrolithotomy (SMP): A new concept in technique and instrumentation. BJU Int 2016;117:655-661.

16. Tefekli A, Ali Karadag M, Tepeler K, et al. Classification of percutaneous nephrolithotomy complications using the modified clavien grading system: Looking for a standard. Eur Urol 2008;53:184-190.

17. Smaldone MC, Corcoran AT, Docimo SG, Ost MC. Endourological management of pediatric stone disease: Present status. J Urol 2009;181:17-28.

18. Hernandez JD, Ellison JS, Lendvay TS. Current trends, evaluation, and management of pediatric nephrolithiasis. JAMA Pediatr 2015;169:964-970.
19. Muslumanoglu AY, Tefekli A, Sarilar O, Binbay M, Altunrende F, Ozkuvanci U. Extracorporeal shock wave lithotripsy as first line treatment alternative for urinary tract stones in children: A large scale retrospective analysis. J Urol 2003;170(6 Pt 1):2405-2408.

20. Koyuncu H, Yencilek F, Erturhan S, Eryildirim B, Sarica K. Clinical course of pediatric urolithiasis: Follow-up data in a long-term basis. Int Urol Nephrol 2011;43:7-13.

21. Bilen CY, Kocak B, Kitirci G, Ozkaya O, Sarikaya S. Percutaneous nephrolithotomy in children: Lessons learned in 5 years at a single institution. J Urol 2007;177:18671871.

22. Ganpule AP, Bhattu AS, Desai M. PCNL in the twenty-first century: Role of microperc, miniperc, and ultraminiperc. World J Urol 2015;33:235-240.

23. Istanbulluoglu MO, Ozturk B, Gonen M, Cicek T, Ozkardes H. Effectiveness of totally tubeless percutaneous nephrolithotomy in selected patients: A prospective randomized study. Int Urol Nephrol 2009;41:541-545.

24. Streem SB, Yost A, Mascha E. Clinical implications of clinically insignificant store fragments after extracorporeal shock wave lithotripsy. J Urol 1996;155:1186-1190.

25. Delvecchio FC, Preminger GM. Management of residual stones. Urol Clin North Am 2000;27:347-354.

26. Rassweiler JJ, Renner C, Chaussy C, Thuroff S. Treatment of renal stones by extracorporeal shockwave lithotripsy: An update. Eur Urol 2001;39:187-199.

27. Turk C, Petrik A, Sarica K, et al. EAU guidelines on interventional treatment for urolithiasis. Eur Urol 2016;69: $475-482$.

Address correspondence to: Guohua Zeng, $M D, P h D$ Department of Urology Minimally Invasive Surgery Center the First Affiliated Hospital of Guangzhou Medical University Guangdong Key Laboratory of Urology Kangda Road $1^{\#}$, Haizhu District Guangzhou 510230

China

E-mail: gzgyzgh@vip.tom.com

\begin{aligned} & \multicolumn{1}{c}{ Abbreviations Used } \\ & CIRF $=$ clinically insignificant residual fragments \\ & $\mathrm{CT}=$ computed tomography \\ & $\mathrm{KUB}=$ kidneys, ureters, and bladder \\ & $\mathrm{LOS}=$ length of stay \\ & $\mathrm{PCNL}=$ percutaneous nephrolithotomy \\ & $\mathrm{RIRS}=$ retrograde intrarenal surgery \\ & $\mathrm{SFR}=$ stone-free rate \\ & $\mathrm{SMP}=$ super-mini percutaneous nephrolithotomy \\ & SWL $=$ shock wave lithotripsy \\ & $\mathrm{UMP}=$ ultra-miniperc \\ & $\mathrm{US}=$ ultrasonography \end{aligned}

